\title{
Missing Values and Indeterminable Values in Fuzzy Relational Compositions
}

\author{
Nhung Cao \\ CE IT4I - IRAFM, University of Ostrava, \\ 30. dubna 22, 70103 Ostrava, Czech Republic, \\ nhung.cao@osu.cz
}

\begin{abstract}
Four-valued logics were developed from three-valued logics aiming at creating tools dealing with missing values and indeterminable values at the same time in computer science. In this paper, we are motivated by the formation of such four-valued logics and construct a new set of operations able to work with both types of such undefined values in partial fuzzy set theory. The operations are built based on the well-known Bochvar algebra and the Sobociński algebra modeling indeterminable values, and the recent Dragonfly algebra purely designed for modeling missing values. Moreover, the operations are established to be compatible with the operations used for elaborating compositions of partial fuzzy relations. Various valid properties of the new operations are then presented, and consequently, the properties of the compositions of partial fuzzy relations constructed based on such operations are implied as well. In the end, an illustrative example is provided to observe the use of the proposed compositions.
\end{abstract}

Keywords: Fuzzy relational compositions, Compositions of partial fuzzy relations, Partial fuzzy set theory, Undefined values, Missing values, Indeterminable values.

\section{Preliminaries}

\subsection{Preliminaries of undefined values}

Three-valued logics were initially proposed in the 1920 s by Łukasiewicz [21]. In the years later, the interest of the logicians on the topic was continually evolving. This can be substantiated by numerous studies regarding the development of the theory $[5,6]$ and various areas of applications such as electronic circuits [25], logic programming [17] or logics for artificial intelligence [26]. The general motivation for forming such logics was to fit better with several practical problems where the statements can be "indeterminable", "unknown", "meaningless", or "inconsistent". Their respective truth values were modelled as undefined values and represented by a dummy value, usually denoted by $\star$.

From the global point of view, $\star$ values are classified into two different types depending on their ability to be redefined. The first type corresponds to the case when truth values are inconsistent, meaningless or indeterminable and there exists no possible way to determine them. The second type corresponds to the case when truth values are missing i.e., they have not been yet established, but can be determined eventually by some ways such as waiting for a certain time, trying with a stronger computer. In computer science, these two types are mentioned as inconsistent information and incompleteness information (cf. [1]). In [2], the first type corresponds to critical errors and the second type corresponds to non-critical errors. In [15], the authors mentioned these two types using the terms of ontological undefined values and epistemic undefined values, respectively. In the sequel, let us denote the first type by using the term "indeterminable values" and the second type by "missing values". Several wellknown three-valued logics were built for modeling each single type such as Bochvar logic, Sobociński logic, McCarthy logic and Kleene logic (cf. [18, 9]).

The development of undefined values did not stop at three-valued logics but upgraded to four-valued logics $[8,20]$. There, the theoretical models were built to create suitable tools that can handle both types of undefined values at the same time. Distinct four-valued logics were constructed in many different ways. They could be formed by a combination of two three-valued logics, for example, the combination of McCarthy logic and Kleene logic was studied in [20], a sort of combina- 
tion of Kleene logic and Priest logic which formed the well-known Belnap four-valued logic was introduced in [8]. Apart from that, four-valued logics which were independently elaborated without combining single logics were studied as well, e.g., the Ferreira four-valued logic [16]. Recently, another so-called four-valued paradefinite logic was addressed [1]. Furthermore, there exist also logics with higher number of elements such as five-valued logic and six-valued logic [19].

\subsection{Preliminaries of operations}

A generalization of three-valued logics to partial fuzzy logics and the formation of the so-called partial fuzzy set theory have been recently approached [7, 23]. There, the operations were elaborated based on the support $L^{\star}=L \cup\{\star\}$ standing for the extension of the support $L$ of a residuated lattice by the dummy value $\star$. In the following, let us fix a residuated lattice $\mathcal{L}=\langle L, \wedge, \vee, \otimes, \rightarrow 0,1\rangle$ where $L=[0,1]$ as the underlying algebraic structure. Note that the operations considered in this paper, which are not attached by any subscript, are operations from the structure $\mathcal{L}$. Now, we briefly recall the definitions of the Bochvar operations, the Sobociński operations and the Dragonfly operations.

Definition 1 [7] The Bochvar operation $c_{B} \in$ $\left\{\wedge_{B}, \vee_{B}, \otimes_{B}, \rightarrow_{B}\right\}, c_{B}: L^{\star} \times L^{\star} \rightarrow L^{\star}$ is represented by the following truth table:

\begin{tabular}{c|c|c|}
$c_{B}$ & $\beta$ & $\star$ \\
\hline$\alpha$ & $\alpha c \beta$ & $\star$ \\
\hline$\star$ & $\star$ & $\star$
\end{tabular}

Definition 2 [7] The Sobociński operation $c_{S} \in$ $\left\{\wedge_{S}, \vee_{S}, \otimes_{S}\right\}, c_{S}: L^{\star} \times L^{\star} \rightarrow L^{\star}$ and the Sobocinski implication $\rightarrow_{S}$ : $L^{\star} \times L^{\star} \rightarrow L^{\star}$ are represented by the following truth tables:

\begin{tabular}{c|c|c|}
$c_{S}$ & $\beta$ & $\star$ \\
\hline$\alpha$ & $\alpha c \beta$ & $\alpha$ \\
\hline$\star$ & $\beta$ & $\star$
\end{tabular},$\quad$\begin{tabular}{r|c|c|}
$\rightarrow_{S}$ & $\beta$ & $\star$ \\
\hline$\alpha$ & $\alpha \rightarrow \beta$ & $\neg \alpha$ \\
\hline$\star$ & $\beta$ & $\star$
\end{tabular}.

The Dragonfly algebra has been approached in [27]. It was motivated by the lower estimation strategy [12]. We recall that on $L^{\star} \backslash\{0,1\}$, the Dragonfly conjunction operations correspond to the Bochvar conjunction operations and the disjunction operations correspond to the Sobociński disjunction operations. The implication operation applying for an element $a \in L$ and the dummy value $\star$ results the value $a$ when $\star$ is applied for the first argument and results $\star$ when $\star$ is applied for the second argument. The operations applying for $\star$ and $\{0,1\}$ in the Dragonfly algebra preserve the results as in case of the Kleene algebra i.e., the ordering $0 \leq \star \leq 1$ is preserved. However, it is adopted by single minor modification, in particular, $\star \rightarrow_{D} \star=1$ although $\star \rightarrow_{K} \star=\star$ in the Kleene case. This modification ensures the preservation of the well-known ordering property in a residuated lattice that $\alpha \rightarrow \beta=1$ if and only if $\alpha \leq \beta$.

Definition 3 [27] The Dragonfly operations $c_{D} \in$ $\left\{\otimes_{D}, \wedge_{D}\right\}, \vee_{D}, \rightarrow_{D}: L^{\star} \times L^{\star} \rightarrow L^{\star}$ are represented by the following truth tables:

\begin{tabular}{c|c|c|c|c|}
$c_{D}$ & $\beta$ & $\star$ & 0 & 1 \\
\hline$\alpha$ & $\alpha c \beta$ & $\star$ & 0 & $\alpha$ \\
\hline$\star$ & $\star$ & $\star$ & 0 & $\star$ \\
\hline 0 & 0 & 0 & 0 & 0 \\
\hline 1 & $\beta$ & $\star$ & 0 & 1
\end{tabular},

\begin{tabular}{r|c|c|c|c|}
$\vee_{D}$ & \multicolumn{1}{|c|}{$\beta$} & $\star$ & 0 & 1 \\
\hline$\alpha$ & $\alpha \vee \beta$ & $\alpha$ & $\alpha$ & 1 \\
\hline$\star$ & $\beta$ & $\star$ & $\star$ & 1 \\
\hline 0 & $\beta$ & $\star$ & 0 & 1 \\
\hline 1 & 1 & 1 & 1 & 1
\end{tabular}

\begin{tabular}{r|c|c|c|c}
$\rightarrow_{D}$ & $\beta$ & $\star$ & 0 & 1 \\
\hline$\alpha$ & $\alpha \rightarrow \beta$ & $\star$ & $\neg \alpha$ & 1 \\
\hline$\star$ & $\beta$ & 1 & $\star$ & 1 \\
\hline 0 & 1 & 1 & 1 & 1 \\
\hline 1 & $\beta$ & $\star$ & 0 & 1
\end{tabular}.

Let us recall [7] two external operations of $\downarrow, \uparrow$ which are defined as follows: $\downarrow \alpha=0$ if $\alpha=\star$ and $\downarrow \alpha=\alpha$ otherwise; $\uparrow \alpha=1$ if $\alpha=\star$ and $\uparrow \alpha=\alpha$ otherwise.

\section{Designing a set of new operations}

\subsection{Fuzzy relational compositions and motivation}

In this paper, we deal with fuzzy relational compositions. They have been intensively studied by Bandler and Kohout in the early 1980s [4] and since then they have become an important topic in fuzzy mathematics. Their applications are various, including e.g., architectures for information processing [3], relational databases [24], or fuzzy inference systems [28]. To shorten the name, let us mention them as standard compositions. For more details regarding their definitions and properties, we may refer to $[14,10]$. It is worth mentioning that the topic is still updated and extended in distinct directions including the recent one on the incorporation of excluding features [13].

As soon as the partial fuzzy set theory was formed, we modified the standard compositions to the compo- 
sitions of partial fuzzy relations [11] to get compatibility with practical situations where the occurrence of the unknown values is rather frequent. In [11], we construct such compositions using a suitable combination of the Bochvar operations and the Sobociński operations. In particular, the compositions applied the Bochvar operations for the conjunction operations and the Sobociński operations for the disjunction operations and the implication operations. The choice of such combination of the operations into the compositions was explained based on an animal classification example (cf. page 5 in [11]).

In addition to the above compositions dealing with indeterminable values, we have proposed the compositions modeling missing values as well [27]. Such compositions were built based on the setting of the Dragonfly algebra. Let us recall that this algebra was designed to reflect the lower estimation strategy [12] which was an initial idea to set up a set of operations not only dealing with missing values but also having compatibility in using operations for modeling compositions of partial fuzzy relations. It should be mentioned that the potential of the compositions was demonstrated in the Dragonflies classification problem [27].

The question is, whether we can build compositions of partial fuzzy relations dealing with both types of undefined values or not. Similar in the case of fourvalued logic, where both types may appear together in a single set of operations, we found that the answer is rather positive.

Another reason to approach compositions of partial fuzzy relations dealing with both types of undefined values is that only a type of undefined values is hard to appear in a practical problem. For example, let us focus on the real example of Dragonflies classification problem where the missing values are most likely to occur. Such values are caused by the lack of the skill or the lack of time to observe of an observer. However, the indeterminable values may also exist as well, e.g., due to the changes in the Dragonflies' features, depending on their living environment or their growth. A visible instance for this case is that the Crocothemis Servilia Dragonfly changes its color from yellow to orange and to red in the process of maturity. Thus, the statement of "Crocothemis Servilia is yellow" has an indeterminable truth value. This example is similar to the typical one that "Bats are warm-blooded" which is inconsistent as bats are warm-blooded in their active period and they are cold-blooded during their hibernation. Such cases are common in nature.

\subsection{Designing new operations}

This section introduces a set of operations dealing with missing values and indeterminable values. We approach to build such operations as follows: when restricting on missing values, the operations correspond to the Dragonfly operations, and when restricting on indeterminable values, the operations correspond to a suitable combination of the Bochvar conjunction operations and the Sobociński disjunction and implication operations. This initial intent is not only to design the operations relating two types of undefined values but generally is to bring operations matching with the choice of operations used for modeling compositions of partial fuzzy relations. Indeed, we have recalled that the Dragonfly operations are appropriate for constructing the compositions dealing missing values [27] and the mentioned combination of the Bochvar operations and the Sobociński operations are appropriate to form the compositions dealing indeterminable values [11].

To distinguish the notations, let us use $\star_{I}$ modeling indeterminable values and $\star_{M}$ modeling missing values. Let $L^{\star_{I}}$ stands for $L \cup\left\{\star_{I}\right\}$ and $L^{\star_{M}}$ stands for $L \cup\left\{\star_{M}\right\}$. Then, let $L{ }^{\circledast}=L \cup\left\{\star_{I}, \star_{M}\right\}=L^{\star_{I}} \cup L^{\star_{M}}$ stands for the extension of $L$ by adding two dummy values $\star_{I}, \star_{M}$.

Thus, the operations on $L^{\circledast}$ can be approached as follows: when restricting on $L^{\star_{M}}$, the operations correspond to the Dragonfly operations and when restricting on $L^{\star} I$, the conjunction operations correspond to the Bochvar operations, the disjunction and the implication operations correspond to Sobociński operations. The important question is, how the operations applying for the value $\star_{I}$ and $\star_{M}$ can be defined. Note that $\star_{M}$ represents for the missing values and it can be found out in some ways and in case of the replacement, the operations will be identical with operations defined on $L^{\star_{I}}$. Thus, the operations applying for $\star_{I}$ and $\star_{M}$ can be approached in the same way as approaching the operations applying for $\star_{I}$ and any element from $L$. In particular, the conjunction of $\star_{I}$ and $\star_{M}$ returns $\star_{I}$ again. The disjunction of the same elements results $\star_{M}$. And for the implication, in both arguments, $\star_{I}$ works as a dummy value in the Sobociński algebra. This determination of $\star_{I}$ and $\star_{M}$ is nothing else but identical to the assumption that $\star_{I}$ prevails over $\star_{M}$. Let us recall that such the assumption was equivalently adopted by Avron and Konikowski in their paper [2] when they studied the combination of the McCarthy algebra and the Kleene algebra.

For the simplicity, we denote the proposed operations by the usual operations adding the subscript " $C$ ". Here, " $C$ " stands for the combination of the Bochvar operations, the Sobociński operations, and the Drag- 
onfly operations.

Definition 4 The operations dealing with missing values and indeterminable values $\Pi_{C} \in\left\{\otimes_{C}, \wedge_{C}\right\}, \vee_{C}$, $\rightarrow_{C}: L^{\circledast} \times L^{\circledast} \rightarrow L^{\circledast}$ are represented by the following truth tables:

\begin{tabular}{r|c|c|c|c|c|}
$\sqcap_{C}$ & $\beta$ & $\star_{I}$ & $\star_{M}$ & 0 & 1 \\
\hline$\alpha$ & $\alpha \sqcap \beta$ & $\star_{I}$ & $\star_{M}$ & 0 & $\alpha$ \\
\hline$\star_{I}$ & $\star_{I}$ & $\star_{I}$ & $\star_{I}$ & $\star_{I}$ & $\star_{I}$ \\
\hline$\star_{M}$ & $\star_{M}$ & $\star_{I}$ & $\star_{M}$ & 0 & $\star_{M}$ \\
\hline 0 & 0 & $\star_{I}$ & 0 & 0 & 0 \\
\hline 1 & $\beta$ & $\star_{I}$ & $\star_{M}$ & 0 & 1
\end{tabular}

\begin{tabular}{r|c|c|c|c|c|}
$\vee_{C}$ & $\beta$ & $\star_{I}$ & $\star_{M}$ & 0 & 1 \\
\hline$\alpha$ & $\alpha \vee \beta$ & $\alpha$ & $\alpha$ & $\alpha$ & 1 \\
\hline$\star_{I}$ & $\beta$ & $\star_{I}$ & $\star_{M}$ & 0 & 1 \\
\hline$\star_{M}$ & $\beta$ & $\star_{M}$ & $\star_{M}$ & $\star_{M}$ & 1 \\
\hline 0 & $\beta$ & 0 & $\star_{M}$ & 0 & 1 \\
\hline 1 & 1 & 1 & 1 & 1 & 1
\end{tabular},

\begin{tabular}{r|c|c|c|c|c|}
$\rightarrow_{C}$ & $\beta$ & $\star_{I}$ & $\star_{M}$ & 0 & 1 \\
\hline$\alpha$ & $\alpha \rightarrow \beta$ & $\neg \alpha$ & $\star_{M}$ & $\neg \alpha$ & 1 \\
\hline$\star_{I}$ & $\beta$ & 1 & $\star_{M}$ & 0 & 1 \\
\hline$\star_{M}$ & $\beta$ & $\star_{M}$ & 1 & $\star_{M}$ & 1 \\
\hline 0 & 1 & $\star_{I}$ & 1 & 1 & 1 \\
\hline 1 & $\beta$ & 0 & $\star_{M}$ & 0 & 1
\end{tabular}.

Furthermore, the negation $\neg C: L^{\circledast} \rightarrow L^{\circledast}$ and the bi-implication $\leftrightarrow_{C}: L^{\circledast} \times L^{\circledast} \rightarrow L^{\circledast}$ are defined as follows for $a, b \in L^{\circledast}$ :

$$
\begin{aligned}
\neg_{C} a & =a \rightarrow_{C} 0, \\
a \leftrightarrow_{C} b & =\left(a \rightarrow_{C} b\right) \wedge_{C}\left(b \rightarrow_{C} a\right) .
\end{aligned}
$$

Let us shortly focus on the position of $\star_{I} \rightarrow_{C} \star_{I}$ in the truth table of the implication. We know that the employment of the Sobociński implication operation on $L^{\star_{I}}$ would lead to $\star_{I} \rightarrow_{C} \star_{I}=\star_{I}$. However, to preserve the well-known ordering property in a residuated lattice that $\alpha \rightarrow \beta=1$ if and only if $\alpha \leq \beta$, we approach to define: $\star_{I} \rightarrow_{C} \star_{I}=1$. It is worth mentioning that such a setting of the implication was approached in the Dragonfly algebra [27]. Moreover, it equivalently holds in many algebras in three-valued logic such as in the Nelson algebra, the Łukasiewicz algebra, the Gödel algebra, or even in the Bochvar external algebra which deals with indeterminable value (cf. [15]). On the other hand, it makes a good sense from the application point of view, e.g., for a set of fuzzy rules, if the antecedent of a rule enters with $\star_{I}$, the rule can be omitted and does not affect on the other rules in the reasoning process.

Remark 1 The proposed operations are formed based on a sort of the combination of three algebras of op- erations with a slight modification for some pairs of elements. This approach is not new as the existence of the combination of the Bochvar algebra, the Sobocinski algebra and the Kleene algebra into a set of operations has been formed in partial fuzzy set theory [22]. In that paper, the authors defined the ordering of the dummy values according to the three algebras to define the operations connecting them.

\subsection{Properties}

This section is devoted to investigating properties of the proposed operations. Let us recall that the ordering $\leq$ on $L^{\circledast}$ is defined such that it reflects the chain $L=[0,1]$ and apart from that, $0 \leq \star_{M} \leq 1, \star_{M}$ is incomparable to any $\alpha \in L \backslash\{0,1\}$, and finally, $\star_{I}$ is not comparable to any $\alpha \in L^{\star M}$.

Lemma 2.1 The following hold for any $a, b, c \in L^{\circledast}$ :

$$
\begin{aligned}
& a \otimes_{C} b \leq a \wedge_{C} b, \\
& a \otimes_{C} b=b \otimes_{C} a, \quad a \wedge_{C} b=b \wedge_{C} a, \\
& a \vee_{C} b=b \vee_{C} a, \\
& a \wedge_{C}\left(b \wedge_{C} c\right)=\left(a \wedge_{C} b\right) \wedge_{C} c, \\
& a \vee_{C}\left(b \vee_{C} c\right)=\left(a \vee_{C} b\right) \vee_{C} c, \\
& a \wedge_{C}\left(b \vee_{C} c\right)=\left(a \wedge_{C} b\right) \vee_{C}\left(a \wedge_{C} c\right), \\
& a \leq b \quad \text { iff } a \rightarrow_{C} b=1, \\
& a \leq b \Rightarrow b \rightarrow_{C} c \leq a \rightarrow_{C} c, \\
& a \leq b \Rightarrow \neg_{C} b \leq \neg_{C} a .
\end{aligned}
$$

Sketch of the proof: The validity of (1)-(9) can be easily checked by making $\star_{I}$ or $\star_{M}$ appearing in the arguments.

Lemma 2.2 Let $\mathcal{L}$ be a residuated lattice such that $\otimes$ is without zero divisors. Then the following porperties hold for any $a, b, c \in L^{\circledast}$ :

$$
\begin{aligned}
a \otimes_{C}\left(b \otimes_{C} c\right) & =\left(a \otimes_{C} b\right) \otimes_{C} c, \\
\left(a \vee_{C} b\right) \otimes_{C} c & =\left(a \otimes_{C} c\right) \vee_{C}\left(b \otimes_{C} c\right) .
\end{aligned}
$$

Sketch of the proof: The properties naturally hold in $L^{\star} I$ and the assumption of $\otimes$ has no zeros divisors leads to the validity of them on $L^{\star_{M}}$ as well.

Lemma 2.3 The following holds for any $a, b, c \in L^{\circledast}$ :

$$
\begin{aligned}
& \downarrow\left(a \otimes_{C}\left(b \otimes_{C} c\right)\right)=\downarrow\left(\left(a \otimes_{C} b\right) \otimes_{C} c\right), \\
& \downarrow\left(\left(a \vee_{C} b\right) \otimes_{C} c\right)=\downarrow\left(\left(a \otimes_{C} c\right) \vee_{C}\left(b \otimes_{C} c\right)\right), \\
& a \leq b \Rightarrow\left(a \otimes_{C} c\right) \leq \downarrow\left(b \otimes_{C} c\right) .
\end{aligned}
$$

Sketch of the proof: On $L^{\star_{I}}$, the equalities in (12)-(13) and the inequality in (14) hold without the help of $\downarrow$. However, on $L^{\star_{M}}$, the operation $\downarrow$ has to be considered for the validity of (12)-(14). 
Lemma 2.4 For any $a, b, c \in L^{\circledast}$ :

$$
\downarrow\left(a \otimes_{C} b\right) \leq \downarrow c \quad \text { iff } \quad \downarrow a \leq \downarrow b \rightarrow_{C} \downarrow c .
$$

Sketch of the proof: Alternately replacing $a, b, c$ by $\star_{M}$ and similarly, by $\star_{I}$ and check the equivalence of both inequalities with help of $\downarrow$.

\section{Compositions of partial fuzzy relations}

This section directly applies the proposed operations to define compositions of partial fuzzy relations and study their properties. By $\mathcal{F}^{\circledast}(U)=\left\{A: U \rightarrow L^{\circledast}\right\}$, we denote a set of all partial fuzzy set on $U$.

Definition 5 Let $X, Y, Z$ be finite non-empty universes and let $R \in \mathcal{F}^{\circledast}(X \times Y), S \in \mathcal{F}^{\circledast}(Y \times Z)$. Then compositions $R \circ_{C} S, R \triangleleft_{C} S, R \triangleright_{C} S$ and $R \square_{C} S \in \mathcal{F}^{\circledast}(X \times Z)$ are defined as follows

$$
\begin{aligned}
& \left(R \diamond_{C} S\right)(x, z)=\bigvee_{y \in Y}\left(R(x, y) \otimes_{C} S(y, z)\right), \\
& \left(R \triangleleft_{C} S\right)(x, z)=\bigwedge_{y \in Y}\left(R(x, y) \rightarrow_{C} S(y, z)\right), \\
& \left(R \triangleright_{C} S\right)(x, z)=\bigwedge_{y \in Y}\left(R(x, y) \leftarrow_{C} S(y, z)\right), \\
& \left(R \square_{C} S\right)(x, z)=\bigwedge_{y \in Y}\left(R(x, y) \leftrightarrow_{C} S(y, z)\right) .
\end{aligned}
$$

The meanings of such compositions are similar to that of the standard compositions (cf. [14]). The only difference is that they may deal with undefined values. Following the previous cases of compositions of partial fuzzy relations incorporating the concept of excluding features (cf. [11, 12]), the proposed compositions may employ with such concept of excluding features as well. The definition is given as follows:

Definition 6 Let $X, Y, Z$ be finite non-empty universes and let $R \in \mathcal{F}^{\circledast}(X \times Y), S, E \in \mathcal{F}^{\circledast}(Y \times Z)$. Then the composition $R{ }^{\circ} S^{`} E \in \mathcal{F}^{\circledast}(X \times Z)$ is defined as follows

$$
\begin{aligned}
\left(R \circ_{C} S^{\prime} E\right)(x, z)= & \bigvee_{y \in Y}\left(R(x, y) \otimes_{C} S(y, z)\right) \otimes_{C} \\
& \neg_{C} \bigvee_{y \in Y}\left(R(x, y) \otimes_{C} E(y, z)\right) .
\end{aligned}
$$

Now, we focus on studying the valid properties of the introduced compositions. Let $R, R_{1}, R_{2} \in \mathcal{F}^{\circledast}(X \times Y)$, $S, S_{1}, S_{2} \in \mathcal{F}^{\circledast}(Y \times Z)$, and $T \in \mathcal{F}^{\circledast}(Z \times U)$.

Proposition 3.1 (Associativity of o) Let $\otimes$ be without zero divisors. Then

$$
\left(R \circ_{C} S\right) \circ_{C} T=R \circ_{C}\left(S \circ_{C} T\right) .
$$

Sketch of the proof: Based on (5) and (10).

Proposition $3.2(\downarrow$-Associativity of $\circ)$

$$
\downarrow\left(\left(R \circ_{C} S\right) \circ_{C} T\right)=\downarrow\left(R \circ_{C}\left(S \circ_{C} T\right)\right) .
$$

Sketch of the proof: Due to (12), (13) and the fact that $\downarrow\left(a \vee_{C} b\right)=\downarrow a \vee_{C} \downarrow b$ for all $a, b \in L^{\circledast}$.

Proposition 3.3 (Antitonicity of the BK-product)

$$
\begin{aligned}
R_{1} \subseteq R_{2} & \Rightarrow R_{1} \triangleleft_{C} S \supseteq R_{2} \triangleleft_{C} S, \\
S_{1} \subseteq S_{2} & \Rightarrow R \triangleright_{C} S_{1} \supseteq R \triangleright_{C} S_{2} .
\end{aligned}
$$

Sketch of the proof: Directly using (8).

Proposition 3.4 ( $\downarrow$-Monotonicity of $\circ)$

$$
\begin{aligned}
R_{1} \subseteq R_{2} & \Rightarrow \downarrow\left(R_{1} \circ_{C} S\right) \subseteq \downarrow\left(R_{2}{ }^{\circ} C S\right), \\
S_{1} \subseteq S_{2} & \Rightarrow \downarrow\left(R \circ_{C} S_{1}\right) \subseteq \downarrow\left(R \circ_{C} S_{2}\right) .
\end{aligned}
$$

Sketch of the proof: Applying (14) and the fact that $\downarrow\left(a \vee_{C} b\right)=\downarrow a \vee_{C} \downarrow b$ for all $a, b \in L^{\circledast}$.

Proposition 3.5 (Union- $\odot$ distributivity) Let $\otimes$ be without zero divisors. Then

$$
\begin{aligned}
\left(R_{1} \cup_{C} R_{2}\right) \circ_{C} S & =\left(R_{1} \circ_{C} S\right) \cup_{C}\left(R_{2} \circ_{C} S\right), \\
R \circ_{C}\left(S_{1} \cup_{C} S_{2}\right) & =\left(R \circ_{C} S_{1}\right) \cup_{C}\left(R \circ_{C} S_{2}\right) .
\end{aligned}
$$

Sketch of the proof: Based on property (11).

Proposition 3.6 ( $\downarrow$-Union-॰ distributivity)

$$
\begin{aligned}
& \downarrow\left(\left(R_{1} \cup_{C} R_{2}\right) \circ_{C} S\right)=\downarrow\left(\left(R_{1} \circ_{C} S\right) \cup_{C}\left(R_{2} \circ_{C} S\right)\right), \\
& \downarrow\left(R \circ_{C}\left(S_{1} \cup_{C} S_{2}\right)\right)=\downarrow\left(\left(R \circ_{C} S_{1}\right) \cup_{C}\left(R \circ_{C} S_{2}\right)\right) .
\end{aligned}
$$

Sketch of the proof: Using property (13) and the fact that $\downarrow\left(a \vee_{C} b\right)=\downarrow a \vee_{C} \downarrow b$ for all $a, b \in L^{\circledast}$.

Proposition 3.7 Let $R^{T}(x, y)=R(y, x)$. Then

$$
\begin{gathered}
\left(R \circ_{C} S\right)^{T}=S^{T} \circ_{C} R^{T}, \\
\left(R \triangleleft_{C} S\right)^{T}=S^{T} \triangleright_{C} R^{T}, \\
\left(R \triangleright_{C} S\right)^{T}=S^{T} \triangleleft_{C} R^{T}, \\
\left(R \square_{C} S\right)^{T}=S^{T} \square_{C} R^{T} .
\end{gathered}
$$

Sketch of the proof: Using that $\otimes_{C}$ is commutative.

\section{Proposition 3.8}

$$
R \square_{C} S=\left(R \triangleleft_{C} S\right) \cap_{C}\left(R \triangleright_{C} S\right) .
$$

Sketch of the proof: Applying the definition of $\leftrightarrow_{C}$ and property (4).

When incorporating with excluding features, the following properties are obtained. 
Proposition 3.9 (Associativity of ०) Let $\otimes$ be without zero divisors. Then

$$
\left(R \circ_{C} S\right) \circ_{C} T^{\prime} E=R \circ_{C}\left(S \circ_{C} T\right)^{\prime}\left(S \circ_{C} E\right) .
$$

Sketch of the proof: Based on Proposition 3.1.

Proposition 3.10 ( $\downarrow$-Monotonicity of ०)

$$
\begin{aligned}
S_{1} \subseteq S_{2} & \Rightarrow \downarrow\left(R \circ_{C} S_{1}^{\prime} E\right) \subseteq \downarrow\left(R \circ_{C} S_{2}^{\prime} E\right), \\
E_{1} \subseteq E_{2} & \Rightarrow \downarrow\left(R \circ_{C} S^{\prime} E_{1}\right) \supseteq \downarrow\left(R \circ_{C} S^{\prime} E_{2}\right) .
\end{aligned}
$$

Sketch of the proof: Due to Proposition 3.4 and the fact that $\downarrow\left(a \otimes_{C} b\right)=\downarrow a \otimes_{C} \downarrow b$.

Proposition 3.11 (Union-o distributivity) Let $\otimes$ be without zero divisors. Then

$$
R \circ_{C}\left(S_{1} \cup_{C} S_{2}\right)^{\prime} E=\left(R \circ_{C} S_{1}^{\prime} E\right) \cup_{C}\left(R \circ_{C} S_{2}^{\prime} E\right) .
$$

Sketch of the proof: Applying Proposition 3.5.

Proposition 3.12 ( $\downarrow$-Union-o distributivity)

$$
\begin{aligned}
& \downarrow\left(R \circ_{C}\left(S_{1} \cup_{C} S_{2}\right)^{\prime} E\right) \\
& =\downarrow\left(\left(R \circ_{C} S_{1}^{\prime} E\right) \cup_{C}\left(R \circ_{C} S_{2}^{\prime} E\right)\right) .
\end{aligned}
$$

Sketch of the proof: Using property (13) and the fact that $\downarrow\left(a \otimes_{C} b\right)=\downarrow a \otimes_{C} \downarrow b$.

\section{Illustrative example and discussion}

In order to observe the behaviour of the introduced compositions, we consider a simple example regarding biological classification problems. Let $X=$ $\left\{x_{1}, \ldots, x_{7}\right\}, Y=\left\{y_{1}, \ldots, y_{12}\right\}$ and $Z=\left\{z_{1}, \ldots, z_{7}\right\}$ are finite sets of animals, features of animals and classes of animals, respectively. The elements in the sets $X, Y$ and $Z$ are considered as follows:

- $x_{1}$ - Rabbit, $x_{2}$ - Flying fox, $x_{3}$ - Prince baskettail, $x_{4}$ - Swallow, $x_{5}$ - Raccon dog, $x_{6}-$ Sunda colugo, $x_{7}-$ Climbing fish;

- $y_{1}$ - animal flies, $y_{2}$ - animal has feathers, $Y_{3}$ animal has fins, $y_{4}-$ animal has hair, $y_{5}-$ animal has teeth, $y_{6}-$ animal climbs (trees), $y_{7}-$ animal lays eggs, $y_{8}-$ animal swims, $y_{9}-$ animal is warmblooded, $y_{10}$ - animal eats plants only (herbivorous), $y_{11}$ - animal is nocturnal (active in night time), $y_{12}-$ animal is skillful on the ground;

- $z_{1}$ - Leporidae, $z_{2}$ - Bats, $z_{3}$ - Dragonfly, $z_{4}$ Bird, $z_{5}-$ Dog, $z_{6}-$ Colugo, $z_{7}-$ Fish.
Partial fuzzy relations $R, S, E$ are considered in Table 1, Table 2 and Table 3. The membership degrees in such relations can be determined based on the following facts. Firstly, there are some pairs of elements that are easily defined for the truth degrees by observing the features of the animals or by learning from the literature that provides information for the dependence of the features and the classes. For example, we know that Rabbit is active between sunset and night so we may determine the truth degree of $R\left(x_{1}, y_{11}\right)$ is 0.5 , similarly, the Sunda colugo is good at climbing trees but not an expert one so we may determine the truth degree of $R\left(x_{6}, y_{6}\right)$ is 0.9 . Secondly, there are some pairs of elements whose truth values are missing or indeterminable due to lacking of information or the pairs of elements contain contradictory pieces of information, respectively. For example, the Flying fox is a kind of bat so it is both warm-blooded and cold-blooded depending on their active and sleeping time, so its relationship with "warm-blooded" is filled in by indeterminable value $\star_{I}$. Similarly, the truth degree of the relationship between feature "animal swims" and the class Dragonfly is assigned by $\star_{I}$, since, the nymphs swim and live underwater that may last as long as five years. In some cases, the replacement by the missing value $\star_{M}$ for the truth values is more appropriate. For instance, we may not know if the Raccoon dog is able to swim or not as some dogs do not swim or swim very badly but we can check for its ability later and thus, its relation with the feature of "animal swims" would be filled in by $\star_{M}$. Similarly, the truth degree for that the Fish class are herbivorous, may be missing in determination as the number of herbivorous fish species remains unknown in the fish population.

\begin{tabular}{c|c|c|c|c|c|c|c|}
$R$ & $y_{1}$ & $y_{2}$ & $y_{3}$ & $y_{4}$ & $y_{5}$ & $y_{6}$ & $y_{7}$ \\
\hline$x_{1}$ & 0 & 0 & 0 & 1 & 1 & 0 & 0 \\
\hline$x_{2}$ & 1 & 0 & 0 & 1 & 1 & $\star_{M}$ & 0 \\
\hline$x_{3}$ & 1 & 0 & 0 & 0.3 & $\star_{M}$ & 0 & 1 \\
\hline$x_{4}$ & 1 & 1 & 0 & 0 & 0 & 0 & 1 \\
\hline$x_{5}$ & 0 & 0 & 0 & 1 & 1 & 0.5 & 0 \\
\hline$x_{6}$ & 0 & 0 & 0 & 1 & 1 & 0.9 & 0 \\
\hline$x_{7}$ & 0 & 0 & 1 & 0 & 0.3 & 0 & 1 \\
\hline
\end{tabular}

\begin{tabular}{c|c|c|c|c|}
$y_{8}$ & $y_{9}$ & $y_{10}$ & $y_{11}$ & $y_{12}$ \\
\hline 0 & 1 & 1 & 0.5 & 1 \\
\hline 0 & $\star_{I}$ & 1 & 1 & 0 \\
\hline$\star_{I}$ & 0 & 0 & 0 & 0 \\
\hline 0 & 1 & 1 & 0 & 0.2 \\
\hline$\star_{M}$ & 1 & 0 & 0 & 1 \\
\hline 0 & 1 & 1 & 1 & 0 \\
\hline 1 & 0 & 0 & $\star_{M}$ & 0.7 \\
\hline
\end{tabular}

Table 1: Relationship of animals and features 


\begin{tabular}{r|c|c|c|c|c|c|c}
$S$ & $z_{1}$ & $z_{2}$ & $z_{3}$ & $z_{4}$ & $z_{5}$ & $z_{6}$ & $z_{7}$ \\
\hline$y_{1}$ & 0 & 1 & 1 & 0.8 & 0 & 0.2 & 0.1 \\
\hline$y_{2}$ & 0 & 0 & 0 & 1 & 0 & 0 & 0 \\
\hline$y_{3}$ & 0 & 0 & 0 & 0 & 0 & 0 & 1 \\
\hline$y_{4}$ & 1 & 1 & 0.1 & 0 & 0.8 & 1 & 0 \\
\hline$y_{5}$ & 1 & 1 & $\star_{M}$ & 0 & 1 & 1 & 0.6 \\
\hline$y_{6}$ & 0 & 0.9 & 0 & 0 & $\star_{I}$ & 0.9 & 0 \\
\hline$y_{7}$ & 0 & 0 & 1 & 1 & 0 & 0 & 0.9 \\
\hline$y_{8}$ & 0 & 0 & $\star_{I}$ & 0.5 & $\star_{I}$ & 0 & 1 \\
\hline$y_{9}$ & 1 & $\star_{I}$ & 0 & 1 & 1 & 1 & 0.1 \\
\hline$y_{10}$ & 0.7 & 0.3 & 0 & $\star_{M}$ & 0 & 1 & $\star_{M}$ \\
\hline$y_{11}$ & 0.8 & 0.9 & $\star_{M}$ & 0.3 & $\star_{I}$ & 1 & $\star_{I}$ \\
\hline$y_{12}$ & 1 & 0 & 0 & 0.4 & 1 & 0 & 0.3 \\
\hline
\end{tabular}

Table 2: Relationship of features and classes

\begin{tabular}{c|c|c|c|c|c|c|c|}
$E$ & $z_{1}$ & $z_{2}$ & $z_{3}$ & $z_{4}$ & $z_{5}$ & $z_{6}$ & $z_{7}$ \\
\hline$y_{1}$ & 1 & 0 & 0 & 0 & 1 & 0.8 & 0.8 \\
\hline$y_{2}$ & 1 & 1 & 1 & 0 & 1 & 1 & 1 \\
\hline$y_{3}$ & 1 & 1 & 1 & 1 & 1 & 1 & 0 \\
\hline$y_{4}$ & 0 & 0 & 0.8 & 1 & 0 & 0 & 1 \\
\hline$y_{5}$ & 0 & 0 & 0 & 1 & 0 & 0 & 0 \\
\hline$y_{6}$ & 1 & 0 & 1 & 1 & 0 & 0 & 1 \\
\hline$y_{7}$ & 1 & 1 & 0 & 0 & 1 & 1 & 0 \\
\hline$y_{8}$ & 1 & 1 & 0 & 0 & 0 & 1 & 0 \\
\hline$y_{9}$ & 0 & 0 & 1 & 0 & 0 & 0 & 0.8 \\
\hline$y_{10}$ & 0 & 0 & 1 & 0 & 1 & 0 & 0 \\
\hline$y_{11}$ & 0 & 0 & 0 & 0.7 & 0 & 0 & 0 \\
\hline$y_{12}$ & 0 & 1 & 1 & 0 & 0 & 1 & 0 \\
\hline
\end{tabular}

Table 3: Relationship regarding excluding features of features and classes

To illustrate the results, let us use the Lukasiewicz algebra as the underlying algebraic structure. Then we have the following compositions, $R \circ_{C} S, R \square_{C} S$ and $R{ }^{\circ} S^{`} E$ :

\begin{tabular}{l|c|c|c|c|c|c|c|}
$R \circ_{C} S$ & $z_{1}$ & $z_{2}$ & $z_{3}$ & $z_{4}$ & $z_{5}$ & $z_{6}$ & $z_{7}$ \\
\hline$x_{1}$ & 1 & 1 & 0.4 & 1 & 1 & 1 & 0.6 \\
\hline$x_{2}$ & 1 & 1 & 1 & 0.8 & 1 & 1 & 0.6 \\
\hline$x_{3}$ & 0.3 & 1 & 1 & 1 & 0.1 & 1 & 0.9 \\
\hline$x_{4}$ & 1 & 1 & 1 & 1 & 1 & 1 & 0.9 \\
\hline$x_{5}$ & 1 & 1 & 0.1 & 1 & 1 & 1 & 0.6 \\
\hline$x_{6}$ & 1 & 1 & 0.1 & 1 & 1 & 1 & 0.6 \\
\hline$x_{7}$ & 0.7 & 0.3 & 1 & 1 & 0.7 & 0.3 & 1 \\
\hline
\end{tabular}

\begin{tabular}{l|c|c|c|c|c|c|c}
$R \square_{C} S$ & $z_{1}$ & $z_{2}$ & $z_{3}$ & $z_{4}$ & $z_{5}$ & $z_{6}$ & $z_{7}$ \\
\hline$x_{1}$ & 0.7 & 0 & 0 & 0 & 0 & 0 & 0 \\
\hline$x_{2}$ & 0 & $\star_{M}$ & 0 & 0 & 0 & 0 & 0 \\
\hline$x_{3}$ & 0 & 0 & $\star_{M}$ & 0 & 0 & 0 & 0 \\
\hline$x_{4}$ & 0 & 0 & 0 & $\star_{M}$ & 0 & 0 & 0 \\
\hline$x_{5}$ & $\star_{M}$ & 0 & 0 & 0 & 0 & 0 & 0 \\
\hline$x_{6}$ & 0 & 0 & 0 & 0 & 0 & 0.8 & 0 \\
\hline$x_{7}$ & 0 & 0 & 0 & 0 & 0 & 0 & $\star_{M}$ \\
\hline
\end{tabular}

\begin{tabular}{l|c|c|c|c|c|c|c|}
$R \circ_{C} S^{\prime} E$ & $z_{1}$ & $z_{2}$ & $z_{3}$ & $z_{4}$ & $z_{5}$ & $z_{6}$ & $z_{7}$ \\
\hline$x_{1}$ & 1 & 0 & 0 & 0 & 0 & 0 & 0 \\
\hline$x_{2}$ & 0 & 1 & 0 & 0 & 0 & 0.2 & 0 \\
\hline$x_{3}$ & 0 & 0 & 0.9 & 0.7 & 0 & 0 & 0.1 \\
\hline$x_{4}$ & 0 & 0 & 0 & 1 & 0 & 0 & 0 \\
\hline$x_{5}$ & 0.5 & 0 & 0 & 0 & 1 & 0 & 0 \\
\hline$x_{6}$ & 0.1 & 1 & 0 & 0 & 0 & 1 & 0 \\
\hline$x_{7}$ & 0 & 0 & 0 & 0 & 0 & 0 & 1 \\
\hline
\end{tabular}

The basic composition $R{ }^{\circ} C S$ classified each sample into too many classes with high membership degrees so, it does not help the classification task. To improve such result, the BK-square product $R \square_{C} S$ is applied, however, the membership degrees are lowered too much so that they give no information for the classification problem. Moreover, the appearance of the missing values in the result brings obstacles for the classification task as well. When the basic composition incorporating the excluding features $R{ }^{\circ}{ }_{C} S^{\wedge} E$ is applied, it brings a promising result. In particular, apart from samples $x_{3}$ - Prince basketail and $x_{6}$ - Sunda colugo which have suspicions belonging to the respective classes $z_{4}$ and $z_{2}$ besides their correct classes, the other samples are classified to their correct classes. Moreover, undefined values are eliminated in the obtained result.

The obtained results demonstrate that the proposed compositions may successfully deal with two types of undefined values. In other words, the formation of the proposed operations brings effectiveness. Of course, to get the real potential of such operations as well as the derived compositions, a practical example is necessary to consider. Let us leave this issue for future work.

The proposed operations were formed based on an appropriate combination of the Dragonfly operations, the Sobociński operations, and the Bochvar operations. This direction may not be unique to set up operations working with both types of undefined values. Indeed, one may think of other directions such as a generalization of a four-valued algebra or a six-valued algebra. However, the question is whether these directions provide operations being suitable to elaborate compositions of partial fuzzy relations or not. We may consider this issue as an open problem, and leave it for future work as well. Here, let the proposed operations serve as an initial approach towards building a method of compositions of partial fuzzy relations, helping to solve classification problems whose data may contain missing and indeterminable values.

\section{References}

[1] O. Arieli, A. Avron, Four-valued paradefinite logics, Studia Logica 105 (6) (2017) 1087-1122. 
[2] A. Avron, B. Konikowska, Proof systems for reasoning about computation errors, Studia Logica 91 (2) (2009) 273-293.

[3] W. Bandler, L. J. Kohout, Relational-product architectures for information processing, Information Sciences 37 (1985) 25-37.

[4] W. Bandler, L. J. Kohout, Semantics of implication operators and fuzzy relational products, International Journal of Man-Machine Studies 12 (1) (1980) 89-116.

[5] H. Barringer, J. H. Cheng, C. B. Jones, A logic covering undefinedness in program proofs, Acta Informatica 21 (3) (1984) 251-269.

[6] J. Beall, Off-topic: A new interpretation of weakkleene logic, The Australasian Journal of Logic $13(6)$.

[7] L. Běhounek, V. Novák, Towards fuzzy partial logic, in: 2015 IEEE International Symposium on Multiple-Valued Logic (ISMVL), IEEE, 2015, pp. 139-144.

[8] N. D. Belnap, A useful four-valued logic, in: Modern uses of multiple-valued logic, Springer, 1977, pp. $5-37$.

[9] D. A. Bochvar, M. Bergmann, On a three-valued logical calculus and its application to the analysis of the paradoxes of the classical extended functional calculus, History and Philosophy of Logic 2 (1-2) (1981) 87-112.

[10] L. Běhounek, M. Daňková, Relational compositions in fuzzy class theory, Fuzzy Sets and Systems 160 (8) (2009) 1005-1036.

[11] N. Cao, M. Štěpnička, Compositions of partial fuzzy relations, in: Information Processing and Management of Uncertainty in Knowledge-Based Systems, (IPMU'18), Vol. 855 of Communications in Computer and Information Science, Springer, 2018, pp. 187-198.

[12] N. Cao, M. Štěpnička, Compositions of partial fuzzy relations employing the lower estimation approach, in: The 10th International Conference on Knowledge and Systems Engineering (KSE 2017), IEEE, HCM, Vietnam, 2018, pp. 146-151.

[13] N. Cao, M. Štěpnička, M. Burda, A. Dolný, Excluding features in fuzzy relational compositions, Expert Systems with Applications 81 (2017) 1-11.

[14] N. Cao, M. Štěpnička, M. Holčapek, Extensions of fuzzy relational compositions based on generalized quantifer, Fuzzy Sets and Systems (2018) $73-98$.
[15] D. Ciucci, D. Dubois, A map of dependencies among three-valued logics, Information Sciences 250 (2013) 162-177.

[16] J. U. Ferreira, A four-valued logic, Computer Science \& Information Technology 71.

[17] M. Fitting, A kripke-kleene semantics for logic programs, J. Log. Program. 2 (4) (1985) 295-312.

[18] M. Fitting, Kleene's logic, generalized, Journal of Logic and Computation 1 (6) (1991) 797-810.

[19] O. N. Garcia, M. Moussavi, A six-valued logic for representing incomplete knowledge, in: MultipleValued Logic, 1990., Proceedings of the Twentieth International Symposium on, IEEE, 1990, pp. 110-114.

[20] B. Konikowska, A four-valued logic for reasoning about finite and infinite computation errors in programs, The Many Sides of Logic 21 (2009) 403-423

[21] J. Lukasiewicz, On 3-valued logic (1920), McCall, S.(ed.) Polish Logic (1967) 16-18.

[22] P. Murinová, M. Burda, V. Pavliska, Undefined values in fuzzy logic, in: Advances in Fuzzy Logic and Technology, Springer, 2017, pp. 604-610.

[23] V. Novák, First steps towards harnessing partial functions in fuzzy type theory, in: International Conference on Information Processing and Management of Uncertainty in Knowledge-Based Systems, Springer, 2018, pp. 736-748.

[24] O. Pivert, P. Bosc, Fuzzy preference queries to relational databases, Imperial College Press, London, 2012.

[25] K. C. Smith, The prospects for multivalued logic: A technology and applications view, IEEE Transactions on Computers (9) (1981) 619-634.

[26] R. Turner, Logics for artificial intelligence, Ellis Horwood Ltd.

[27] M. Štěpnička, N. Cao, L. Běhounek, M. Burda, A. Dolný, Missing values and dragonfly operations in fuzzy relational compositions, International Journal of Approximate Reasoning submitted.

[28] M. Štěpnička, B. Jayaram, Interpolativity of atleast and at-most models of monotone fuzzy rule bases with multiple antecedent variables, Fuzzy Sets and Systems 297 (2016) 26-45. 